\title{
The role of dietary fat in adipose tissue metabolism
}

\author{
Alfredo Fernández-Quintela, Itziar Churruca and Maria Puy Portillo* \\ Department of Nutrition and Food Science, University of País Vasco, Paseo de la Universidad 7, \\ 01006 Vitoria, Spain
}

Submitted December 2006: Accepted April 2007

\begin{abstract}
Energy intake and expenditure tend on average to remain adjusted to each other in order to maintain a stable body weight, which is only likely to be sustained if the fuel mix oxidised is equivalent to the nutrient content of the diet. Whereas protein and carbohydrate degradation and oxidation are closely adjusted to their intakes, fat balance regulation is less precise and that fat is more likely to be stored than oxidised.

It has been demonstrated that dietary fatty acids have an influence not only on the fatty acid composition of membrane phospholipids, thus modulating several metabolic processes that take place in the adipocyte, but also on the composition and the quantity of different fatty acids in adipose tissue. Moreover, dietary fatty acids also modulate eicosanoid presence, which have hormone-like activities in lipid metabolism regulation in adipose tissue.

Until recently, the adipocyte has been considered to be no more than a passive tissue for storage of excess energy. However, there is now compelling evidence that adipocytes have a role as endocrine secretory cells. Some of the adipokines produced by adipose tissue, such as leptin and adiponectin, act on adipose tissue in an autocrine/paracrine manner to regulate adipocyte metabolism. Furthermore, dietary fatty acids may influence the expression of adipokines.

The nutrients are among the most influential of the environmental factors that determine the way adipose tissue genes are expressed by functioning as regulators of gene transcription. Therefore, not only dietary fat amount but also dietary fat composition influence adipose tissue metabolism.
\end{abstract}

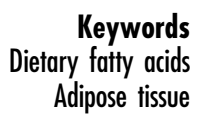

\section{Energy and macronutrient balances}

Although quite variable from day to day, energy intake and energy expenditure tend to remain adjusted to each other on average in order to maintain a stable body weight. Such a steady state is likely to be sustained only if the fuel mix oxidised by the body is equivalent, in terms of its average composition, to the nutrient content of the diet consumed, so that protein balance, carbohydrate balance and fat balance are all achieved. As a result, long periods of weight stability imply not only that energy intake and energy expenditure are equivalent on average but also that the composition of the fuel mix oxidised is equal on average to the composition of the diet. Consequently, it can be stated that spontaneous adjustment of the rate of oxidation of a substrate to its rate of intake is obviously a major factor facilitating maintenance of substrate balance ${ }^{1,2}$.

The rate of aminoacid oxidation and degradation is adjusted to protein intake, so that stable protein content is maintained. The rate of glucose disposal is also closely adjusted to carbohydrate intake because body glycogen reserves are small compared to glucose turnover, not being much larger than the amount of carbohydrate usually consumed and used in one day. Although the conversion of excess carbohydrate into fat can be proposed as a pathway for the disposal of carbohydrates consumed in excess, lipogenesis from carbohydrate is extremely scarce in human adipose tissue. Given that the rates of amino acid and carbohydrate oxidation are determined primarily by protein and carbohydrate intakes, the rate of fat oxidation must be set largely by the gap between total energy expenditure and energy intake in the form of protein and carbohydrates, rather than by the amount of fat consumed on a given day ${ }^{3}$. As a result, oxidation of fat cannot be expected to be determined by fat intake. This suggests that the regulation of fat balance is less precise and that fat is more likely to be stored than oxidised. Consequently, because fat intake does not promote fat oxidation, any excess of fat in the everyday diet is stored and contributes to increase the adipose tissue mass $^{4}$. 
When the percentage of fat in the everyday diet is high, an imbalance between fat intake and oxidation occurs and the adipose tissue mass increases until fat cell hypertrophy and hyperplasia are such that the overall rate of lipolysis in adipose tissue increases the amount of free fatty acids, which stimulate fat oxidation. Thus, stimulation of fat oxidation only occurs when the adipose tissue mass is enlarged. The degree of expansion of the adipose tissue mass required depends on many factors, among which inherited traits can be particularly powerful 5 . Differences in susceptibility to obesity are greatly affected by the influence of inherited differences on an individual propensity to use free fatty acids relative to glucose.

In western societies, with a high dietary fat content, a substantial expansion of adipose tissue mass occurs before the use of fat reaches a rate commensurate with the dietary fat content. This phenomenon may explain the high prevalence of obesity in developed societies, where the constant availability of high desirable foods tends to prevent spontaneous maintenance of reduced glycogen levels ${ }^{5}$. Because errors in fat balance do not bring about the same prompt self-correcting responses as those that operate spontaneously to ensure the maintenance of protein and carbohydrate balances, it appears that conscious efforts to avoid accumulation of fat are likely to be most effective if directed at limiting its consumption ${ }^{1,6}$.

This part of the review has pointed out the influence of dietary fat amount on lipid metabolism, and thus on body mass accumulation. But it is important to emphasise that not only dietary fat amount but also dietary fat composition has an influence on adipose tissue metabolism as described in the following paragraphs.

\section{Fatty acid composition of membrane phospholipids}

Plasma membrane consists of a permeable phospholipid bilayer containing cholesterol and proteins. Phospholipids possess a hydrophilic head located to the outer face of the membrane and a hydrophobic tail in the inner part. Fatty acid comprising membrane phospholipids, which depend on the dietary fat composition, can be saturated or unsaturated, the former having a straight conformation and the latter have a kink in the tail because of a cis double bond. Due to these conformations, saturated fatty acids appear more packed together in the cell membrane, and in turn, more arranged than the unsaturated fatty acids do, affecting some physicochemical membrane properties such as fluidity (Fig. 1).

The presence of polyunsaturated fatty acids in the lipid bilayer contributes to increased membrane fluidity; in contrast, saturated fatty acids are rigidifying molecules, because of the absence of double bounds. Consequently, the $\mathrm{P}: \mathrm{S}$ ratio (polyunsaturated: saturated fatty acids) is known to be representative of the contribution of fatty acids to membrane fluidity.

Membrane fluidity is a key factor in biological terms. Some transport systems (i.e. GLUT-4 glucose transporter), receptors (i.e. $\beta$-adrenergic, insulin receptors)

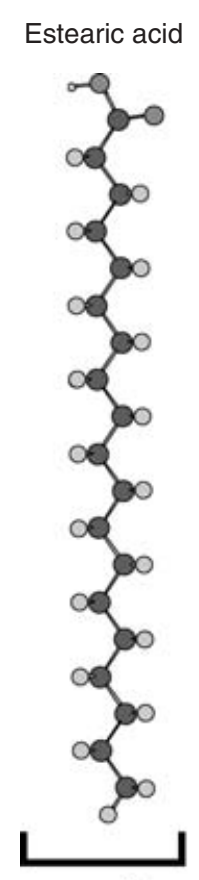

$0,87 \AA$
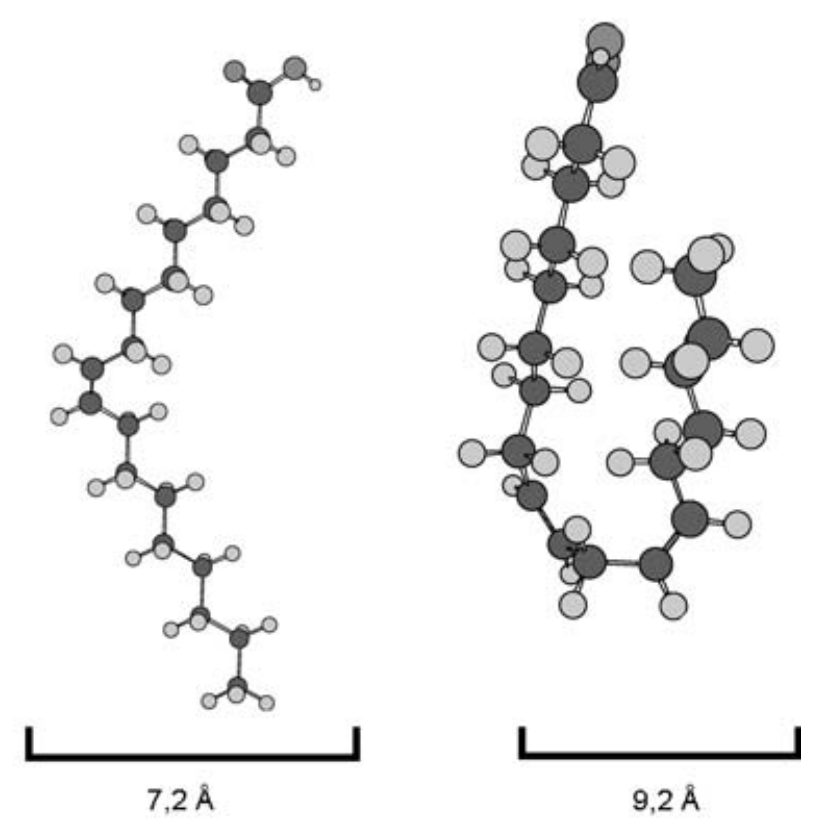

Fig. 1 Structure of saturated (esteraric acid), monounsaturated (oleic acid) and polyunsaturated (linoleic acid) fatty acids 
and enzymatic activities (adenylate cyclase) can be altered when changes in membrane viscosity, an inverse parameter of membrane fluidity, exceed a critical threshold ${ }^{7}$.

It has been demonstrated that dietary fatty acids influence the fatty acid composition of cellular and subcellular membrane phospholipids, and thus can modulate several metabolic processes that take place in the adipocyte ${ }^{7-11}$. The insulin receptor is located embedded in the plasma membrane bilayer and appears to be sensitive to the surrounding lipid environment. The amount of insulin bound and the translocation of GLUT4 transporter induced by this hormone increases with the content of polyunsaturated fatty acids in membrane phospholipids. By contrast, insulin binding decreases with increasing content of saturated and monounsaturated fatty acids ${ }^{12,13}$. With regard to $\beta$-adrenergic-mediated lipolysis, several studies have demonstrated a significant correlation between adipocyte membrane fluidity and the affinities of the $\beta$-adrenergic receptors and have concluded that saturated fat reduces lipolytic activity in adipocytes when compared with unsaturated fat ${ }^{14,15}$.

\section{Selective mobilisation of fatty acids from adipose tissue}

Triacylglycerols, found mainly in adipose tissue, represent the most important lipid store of the body ${ }^{16}$. Adipose tissue triacylglycerols contain a complex mixture of fatty acids that largely reflects the dietary intake of fatty acids that are not synthesised de novo via lipogenesis ${ }^{17}$. Thus, dietary fatty acids are an important source of adipose fatty acids and have a significant effect upon both the composition and the quantity of different fatty acids in adipose tissue ${ }^{18,19}$. These triacylglycerols exist in a dynamic state: they are continuously formed by the influx of fatty acids from the triacylglycerol-rich plasma lipoproteins, but are also continuously hydrolysed to efflux fatty acids into the plasma bound to albumin.

Under conditions of stimulated lipolysis, fatty acids stored as triacylglycerols in adipose tissue are selectively mobilised. The mobilisation of individual fatty acids is not proportional to their content in adipose tissue but rather influenced by their molecular structure. It depends on chain length, unsaturation and cis positional isomerism: it is correlated positively with unsaturation and negatively with the chain length of the fatty acids ${ }^{20-23}$. That being so, saturated fatty acids are mobilised the least and polyunsaturated fatty acids the most, monounsaturated fatty acids remaining intermediate. Highly mobilised fatty acids include those with 16-20 carbon atom chains and 4-5 double bounds, whereas weakly mobilised fatty acids include those with 20-24 carbon atom chains and 0-1 double bond.

In order to explain this selective release of fatty acid, several hypotheses have been proposed and tested.
One of these theories refers to the potential specificity of hormone-sensitive lipase (HSL), the rate-limiting enzyme in the lipolytic cascade, according to fatty acid structure. Nevertheless, Raclot et al. ${ }^{24}$ demonstrated that the release of fatty acids by HSL was only slightly affected by their degree of unsaturation. They concluded that the selectivity of fatty acid hydrolysis by HSL does not fully account for the selective pattern of fatty acid mobilisation.

The catabolic process of apolar lipids by most lipases, including HSL, is closely related to the lipid-water interface ${ }^{25,26}$, where only small amounts of substrate are available to the enzyme. It has been reported that triacylglycerols enriched in polyunsaturated fatty acids display a higher polarity ${ }^{27}$, and thus a higher partition coefficient between an interfacial phase and an apolar phase than triacylglycerols enriched in saturated fatty acids $^{25}$. Thus, at the lipid-water interface, polyunsaturated fatty acids will be more accessible to HSL for hydrolysis, and consequently the relative amount of these fatty acids released will be greater than that of saturated fatty acids ${ }^{21}$.

HSL interacts with an intracellular fatty acid-binding protein, called ALBP, which is highly expressed in adipocytes. Its interaction with the N-terminal domain of HSL facilitates raid shuttling of the fatty acids out of the adipocyte, thus preventing the deleterious effect of fatty acid accumulation during lipolysis ${ }^{24,28}$. As a result, the differential mobilisation of adipose tissue fatty acids may also result from a differential transfer of free fatty acids into the plasma. Moreover, binding to albumin in plasma may also differ for different fatty acids with polyunsaturated having greater affinity for the two tight-binding sites of albumin.

Implications of selective release of fatty acids in physiology and health could exist in the modulation of the supply of biologically active fatty acids to specific tissues.

\section{Eicosanoid and adipokine production}

Arachidonic acid, synthesised from linoleic acid 18:2n-6 and eicosapentaenoic acid, synthesized from linolenic acid (18:3n-3) are the precursors for eicosanoids, $n-6$ and $n-3$ eicosanoids, respectively, including prostaglandins, prostacyclins, leucotrienes, and thromboxanes, via metabolic pathways regulated by cyclo-oxigenase and lipo-oxigenase. Eicosanoids have hormone-like activities and so play important roles in the regulation of several functions and metabolic processes in the body, some of which refer to lipid metabolism in adipose tissue ${ }^{29-32}$. Thus, it has been shown that prostaglandin E2 (PGE2) stimulates leptin release and inhibits basal lipolysis over 24-hour incubation of mouse adipose tissue in primary culture ${ }^{33}$. With regard to reduction in lipolysis, PGE2 produces an elevation of protein Gi activity in adipocyte membrane that leads to inhibition of adenylate cyclase and thus to a reduced $\mathrm{AMP}_{\mathrm{C}}$ level. Consequently, 
HSL is not activated and lipid mobilisation is inhibited. Moreover, eicosanoids are involved in the regulation of several important functions and processes, such as vascular homeostasis, macrophage chemiotaxis and inflammation ${ }^{34}$.

Since these two fatty acid series are metabolised following common metabolic pathways, competence phenomena take place, and as a consequence, depending on the dietary lipid profile, proportions between $n-6$ and $n-3$ derived eicosanoids will change. The biological effects promoted by two eicosanoid series are different, being, depending on the eicosanoid, opposite (PGI2 hypotensor vs. PGF2 hypertensor) or active/non-active forms (PGE2 lypolitic action vs. PGE3 non-lypolitic action). Thus, it can be argued that dietary fatty acid profile is involved in the regulation of the aforementioned functions ${ }^{35,36}$.

Until recently, the adipocyte has been considered to be only a passive tissue for the storage of excess energy in the form of triacylglycerols. However, there is now compelling evidence that adipocytes act as endocrine secretory cells. It has been shown that several hormones, growth factors and cytokines are actually expressed in white adipose tissue ${ }^{37}$. Moreover, cells from the stromal-vascular fraction in adipose tissue also produce this kind of molecules. Some of the adipokines produced by adipose tissue, such as leptin, act in adipose tissue in an autocrine/paracrine manner to regulate adipocyte metabolism. By contrast, others, on their secretion into the bloodstream, act as endocrine signals at multiple distant sites to regulate energy homeostasis. Thus, adiponectin stimulates fatty acid oxidation in skeletal muscle and liver leading to a decreased availability of fatty acids to be stored in adipose tissue as triacylglycerols ${ }^{38}$.

Fatty acids may influence the expression of adipokines like leptin and resistin directly by interaction with transcription factors, or indirectly via unknown mechanisms possibly linked to fatty acid oxidation, synthesis or storage $^{39}$. It has been reported that dietary monounsaturated and polyunsaturated fatty acids may modify plasma leptin concentration in human, rat and mice, by means of a decrease in leptin gene expression both in vivo and in vitro, mediated by mechanisms associated with reduced PPAR $\gamma$ and SREBP-1 gene expression ${ }^{40,41}$. Moreover, it has been shown that resistin is inhibited by polyunsaturated fatty acids. Thus, arachidonic acid and eicosapentaenoic acid reduce resistin mRNA levels in cultured 3T3-L1 adipocytes ${ }^{42}$.

\section{Adipose tissue gene expression}

The development of obesity involves a multitude of gene products, including proteins associated with lipid synthesis and oxidation, thermogenesis and cell differentiation. The genes encoding these proteins are in essence the blueprints that have been inherited from our parents. However, what determines the way these genes are expressed is largely dictated by a collection of environmental factors. The nutrients we consume are among the most influential of these environmental factors. They govern the tissue content of different proteins by functioning as regulators of gene transcription ${ }^{43}$.

Polyunsaturated fatty acids suppress the transcription of genes encoding for lipogenic enzymes (i.e. fatty acid synthase, acetyl-CoA carboxylase and stearoyl CoA desaturase) in adipose tissue and liver. They simultaneously induce the transcription of genes encoding proteins involved in lipid oxidation (carnitine palmitoyltransferase and acyl CoA oxydase), and fatty acid transport (L-FABP) in liver, in lipid transport proteins (FABP and aP2), fatty acid transporters (CD36, lipoprotein lipase) and adipokines (adiponectin and tumoral necrosis factor alpha (TNF- $\alpha$ ) in white adipose tissue and in thermogenesis in brown adipose tissue ${ }^{44-46}$.

Gene transcription activation is mediated by peroxisome proliferator-activated receptors (PPARs). Many mono- and polyunsaturated fatty acids bind directly to $\operatorname{PPAR} \alpha$ at physiological concentrations. In contrast to $\operatorname{PPAR} \alpha$, mainly expressed in oxidative organ and tissues, PPAR $\gamma$ mainly expressed in adipose tissue, have a preference for very unsaturated over mono- and polyunsaturated fatty acids. Some eicosanoids derived from unsaturated fatty acids are also considered PPAR agonists $^{45,47}$. PPARs mediate gene activation through dimerisation with retinoic X receptor (complex RXR-9, cis-retinoic acid) and binding to cis-acting regulatory elements up-stream of the promoter regions in target genes (Fig. 2).

Supression of lipogenic enzyme gene expression induced by polyunsaturated fatty acids does not directly involve PPARs, but rather reflects changes in the expression and nuclear localisation of the transcription factor, sterol-regulatory element-binding protein-1 (SREBP-1).

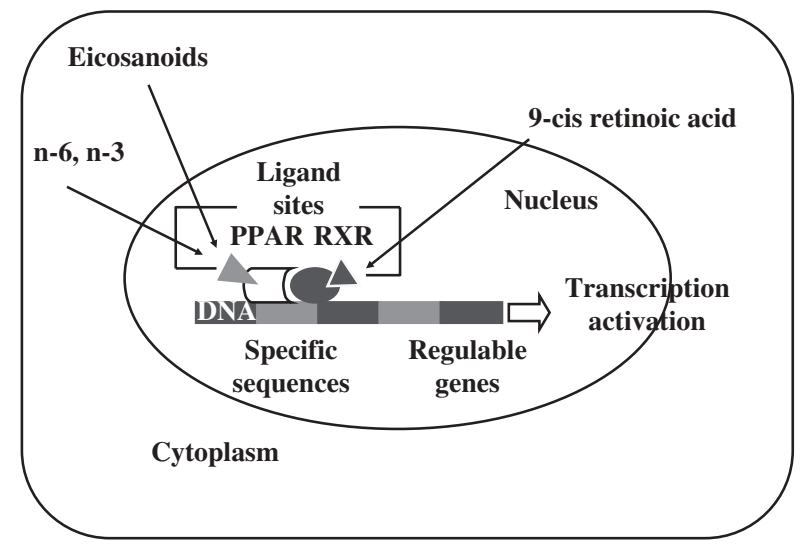

Fig. 2 Induction of gene transcription by peroxisome proliferatoractivated receptors (PPAR) activation 


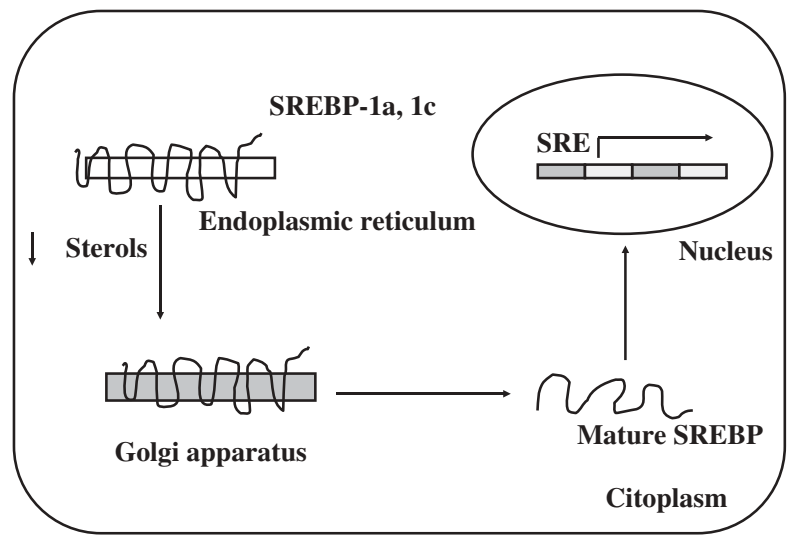

Fig. 3 Induction of gene transcription by sterol regulatory element-binding proteins (SREBP)

SREBPs are synthesised as large proteins and inserted into the endoplasmic reticulum membrane. When cellular sterol level drops, SREBP moves to Golgi apparatus where it undergoes two proteolytic events, giving rise to the smaller mature form of the protein. The mature protein then transits to the nucleus, where it binds to cis elements, termed sterol regulatory elements (SRE), in the promoters of target genes and induces transcription of a variety of genes involved in triacylglycerol and cholesterol metabolism ${ }^{46}$. It has been reported that the ingestion of safflower oil, rich in linoleic acid (18:2 n-6), or fish oil reduces the membrane content of the SREBP-1 precursor and that this in turn leads to a reduction in the nuclear content of SREBP-1 (Fig. 3). The decrease in this transcriptional factor is accompanied by a comparable decrease in the transcription of lipogenic enzymes. Unlike polyunsaturated fatty acids, saturated and monounsaturated fatty acids have no effect on the nuclear content of SREBP-1 or on lipogenic gene expression ${ }^{48-50}$.

\section{Acknowledgements}

Conflict of interest: The authors had no conflicts of interest to report.

Authorship responsibilities: The author's responsibilities were as follows: M.P.P. provided the idea for the manuscript; all the authors contributed the draft and critical revision of the manuscript.

Acknowledgements: The authors are grateful to Dr Gloria Rubiales for preparation of the molecular structures.

\section{References}

1 Flatt JP. Dietary fat, carbohydrate balance and weight maintenance: effects of exercise. American Journal of Nutrition 1987; 45: 296-306.

2 Flatt JP. Importance of nutrient balance in body weight regulation. Diabetes Metabolism Review 1988; 4: 571-81.
3 Flatt JP. Use of storage of carbohydrate and fat. American Journal of Nutrition 1995; 61(Suppl.): 952S-9S.

4 Thomas CD, Peters JC, Reed GW, Abumrad NN, Sun M, Hill JO. Nutrient balance and energy expenditure during ad libitum feeding of high-fat and high-carbohydrate diets in humans. American Journal of Clinical Nutrition 1992; 55 : 934-42.

5 Flatt JP. Glycogen levels and obesity. International Journal of Obesity 1996; 20: S1-S11.

6 Hill JO, Peters JC, Reed GW, Schlundt DG, Sharp T, Greene HL. Nutrient balance in humans: effects of diet composition. American Journal of Clinical Nutrition 1991; 54: 10-17.

7 Cestaro B, Cazzola R, Rondanelli M, Ferrari E. Membrane fluidity and obesity: the correct food approach. Minerva Gastroenterology Dietology 2001; 47: 181-5.

8 Murphy MG. Dietary fatty acids and membrane protein function. Journal of Nutritional Biochemistry 1990; 1: 68-79.

9 Clandinin MT, Cheema S, Field CJ, Garg ML, Venkatraman J, Clandinin TR. Dietary fat: exogenous determination of membrane structure and function. FASEB Journal 1991; 5: 2761-9.

10 Sardesai VM. Nutritional role of polyunsaturated fatty acids. Journal of Nutritional Biochemistry 1992; 3: 154-66.

11 Ibrahim A, Natrajan S, Ghafoorunissa R. Dietary fatty acids alter adipocyte plasma membrane fatty acid composition and insulin sensitivity in rats. Metabolism 2005; 54: 240-6.

12 Berlin E, Bathena SJ, Kliman PG, Revett K. Effect of saturation of dietary lipids on insulin receptors and membrane fluidity in rabbit erythrocytes. Nutrition Reports International 1989; 39: 367-81.

13 Manco M, Calvani M, Mingrone G. Effects of dietary fatty acids on insulin sensitivity and secretion. Diabetes Obesity and Metabolism 2004; 6: 402-13.

14 Awad AB, Cahttopadhay JP. Effect of dietary saturated fatty acids on hormone-sensitive lipolysis in rat adipocytes. Journal of Nutrition 1986; 116: 1088-94.

15 Matsuo T, Sumida H, Suzuki M. Beef tallow decreases beta-adrenergic receptor binding and lipolytic activities in different adipose tissues of rat. Metabolism 1995; 44 : 1271-7.

16 Coppack SW, Jensen MD, Miles JM. In vivo regulation of lipolysis in humans. Journal of Lipid Research 1994; 35: 177-93.

17 Field CJ, Clandinin MT. Modulation of adipose tissue fat composition by diet: a review. Nutrition Research 1984; 4 : 743-55.

18 Mougios V, Kotzamanidis C, Koutsari C, Atsopardis S. Exercise-induced changes in the concentration of individual fatty acids and triacylglycerols of human plasma. Metabolism: Clinical and Experimental 1995; 44: 681-8.

19 Garaulet M, Hernandez-Morante JJ, Lujan J, Tebar FJ, Zamora S. Relationship between fat cell size and number and fatty acid composition in adipose tissue from different fat depots in overweight/obese humans. International Journal of Obesity 2006; 30: 899-905.

20 Raclot T, Groscolas R. Differential mobilization of white adipose tissue fatty acids according to chain length, unsaturation and positional isomerism. Journal of Lipid Research 1993; 34: 1515-26.

21 Raclot T, Groscolas R. Selective mobilization of adipose tissue fatty acids during energy depletion in the rat. Journal of Lipid Research 1995; 36: 2164-73.

22 Connor WE, Lin DS, Colvis C. Differential mobilization of fatty acids from adipose tissue. Journal of Lipid Research 1996; 37: 290-8.

23 Raclot $\mathrm{T}$, Langin D, Lafontan M, Groscolas R. Selective release of human adipocyte fatty acids according to molecular structure. Biochemical Journal 1996; 324: 911-15. 
24 Raclot T, Holm C, Langin D. Fatty acid specificity of hormone-sensitive lipase: implication in the selective hydrolysis of triacylglycerols. Journal of Lipid Research 2001; 42: 2049-57.

25 Hauton JC, Lafont H. Lipid biodynamics: new perspectives. Biochimie 1987; 69: 177-204.

26 Derewenda ZS, Sharp AM. News from the interface: the molecular structures of triacylglyceride lipases. Trends in Biochemical Sciences 1993; 18: 20-5.

27 Perona JS, Portillo MP, Macarulla MT, Tueros AI, RuízGutiérrez V. Influence of different dietary fats on triacylglycerol deposition in rat adipose tissue. British Journal of Nutrition 2000; 84: 765-74.

28 Shen WJ, Sridhar K, Bernlohr DA, Kraemer FB. Interaction of rat hormone-sensitive lipase with adipocyte lipid-binding protein. Proceedings of the National Academy of Sciences USA 1999; 96: 5528-32.

29 Richelsen B. Release and effects of prostaglandins in adipose tissue. Prostaglandins Leukotrienes and Essential Fatty Acids 1992; 47: 171-8.

30 Chatzipanteli K, Rudolph S, Axelrod L. Coordinate control of lipolysis by prostaglandin E2 and prostacyclin in rat adipose tissue. Diabetes 1992; 41: 927-35.

31 Vassaux G, Gaillard D, Darimont C, Ailhaud G, Negrel R. Differential response of preadipocytes and adipocytes to prostacyclin and prostaglandin E2: physiological implications. Endocrinology 1992; 131: 2393-8.

32 Girouard H, Savard R. The lack of bimodality in the effects of endogenous and exogenous prostaglandins on fat cell lipolysis in rats. Prostaglandins and Other Lipid Mediators 1998; 56: 43-52.

33 Fain JM, Leffler CW, Bahouth SW, Rice AM, Rivkees SA. Regulation of leptin release and lipolysis by PGE2 in rat adipose tissue. Prostaglandins and Other Lipid Mediators 2000; 62: 343-50.

34 Bathena SJ. Relationship between fatty acids and the endocrine and neuroendocrine system. Nutritional Neuroscience 2006; 9: 1-10.

35 Zamaria N. Alteration of polyunsaturated fatty acid status and metabolism in health and disease. Reproduction, Nutrition, Development 2004; 44: 273-82.

36 Wijendran V, Hayes KC. Dietary n-6 and n-3 fatty acid balance and cardiovascular health. Annual Review of Nutrition 2004; 24: 597-15.

37 Frübeck G, Gómez-Ambrosi J, Muruzábal FJ, Burrelli MA. The adipocyte: a model for integration of endocrine and metabolic signalling in energy metabolism regulation.
American Journal of Physiology Endocrinolgy and Metabolism 2001; 280: E827-41.

38 Kim S, Moustaid-Moussa N. Secretory, endocrine and autocrine/paracrine function of the adipocyte. Journal of Nutrition 2000; 130: 3110S-5S.

39 Drevon CA. Fatty acids and expression of adipokynes. Biochimica et Biphysica Acta 2005; 1740: 287-92.

40 Reseland JE, Haugen F, Hollung K, Solvoll K, Halvorsen B, Brude IR, et al. Reduction of leptin gene expression by dietary polyunsaturated fatty acids. Journal of Lipid Research 2001; 42: 743-50.

41 Rodríguez VM, Picó C, Portillo MP, Macarulla MT, Palou A. Dietary fat source regulates $o b$ gene expresión in white adipose tissue of rats under hyperphagic feeding. British Journal of Nutrition 2002; 87: 427-34.

42 Haugen F, Zahid KT, Dalen K, Hollung K, Nebb HI, Drevon CA. Resistin expression in 3T3-L1 adipocytes is reduced by arachidonic acid. Journal of Lipid Research 2005; 46: 143-53.

43 Clarke SD. Polyunsaturated fatty acid regulation of gene transcription: a mechanism to improve energy balance and insulin resistance. British Journal of Nutrition 2000; 83(Suppl. 1): S59-66.

44 Kersten S. Peroxisome proliferator activated receptors and obesity. European Journal of Pharmacology 2002; 440: 223-34.

45 Khan SA, Vanden Heuvel JP. Role of nuclear receptors in the regulation of gene expression by dietary fatty acids. Journal of Nutritional Biochemistry 2003; 14: 554-67.

46 Sampath H, Ntambi JM. Polyunsaturated fatty acids regulation of genes of lipid metabolism. Annual Review of Nutrition 2005; 25: 317-40.

47 Deckelbaum RJ, Worgali TS, Seo T. n-3 Polyunsaturated acids and gene expression. American Journal of Clinical Nutrition 2006; 83(Suppl.): 1520S-2S.

48 Osborne TF. Sterol regulatory element-binding proteins (SREBPS): key regulators of nutritional homeostasis and insulin action. The Journal of Nutritional Biochemistry 2000; 275: 32379-82.

49 Shimano H. Sterol regulatory element-binding proteins (SREBPs): transcriptional regulators of lipid synthetic genes. Progress in Lipid Research 2001; 40: 439-52.

50 Horton JD, Goldstein JL, Brown MS. SREBPs: activators of the complete program of cholesterol and fatty acid synthesis in the liver. Journal of Clinical Investigaction 2002; 109: $1125-31$. 